\title{
Role of Inferior Vena Cava Filter Retrieval in Patients on Chronic Anticoagulation Therapy
}

Savannah Fletcher, $M D^{1, *}$

Adam Plotnik, $M D^{1}$

Ravi N. Srinivasa, MD, FSIR ${ }^{1}$

Jeffrey Forris Beecham Chick, MD, MPH, FCIRSE, FSVM ${ }^{2}$ John M. Moriarty, MD, FSIR ${ }^{1}$

\author{
Address \\ *,1Division of Interventional Radiology, Department of Radiology, University of \\ California Los Angeles, 757 Westwood Plaza, Los Angeles, CA, 90024, USA \\ Email: sfletcher@mednet.ucla.edu \\ ${ }^{2}$ Division of Interventional Radiology, Department of Radiology, University of \\ Washington, Seattle, WA, USA
}

Published online: 25 January 2021

(C) The Author(s) 2021

This article is part of the Topical Collection on Vascular Disease

Keywords Inferior vena cava filter - Pulmonary embolism - Deep venous thrombosis - Extended anticoagulant therapy

\section{Abstract}

Purpose of review Describe the role of inferior vena cava filter (IVCF) retrieval in patients on chronic anticoagulation given the overlap of these treatment options in the management of patients with venous thromboembolic disease.

Recent findings Despite the increase in IVCF retrievals since the Food and Drug Administration safety communications in 2010 and 2014, retrieval rates remain low. Previous studies have shown that longer filter dwell times are associated with greater risk for filter complications and more difficulty with filter retrievals. Recent findings suggest that complications are more frequent in the first 30 days after placement.

Summary The decision to retrieve an optional IVCF is individualized and requires diligent follow-up with consistent re-evaluation of the need for the indwelling IVCF, particularly in those on long-term anticoagulation therapy. 


\section{Introduction}

Venous thrombosis is a cause of mortality in the USA with an estimated 1-2 events per 1000 individuals per year and attributable deaths of over 300,000 per year [1]. Pulmonary embolism (PE) carries an 18-fold higher risk of early death when compared to patients with deep venous thrombosis (DVT) alone [2]. Approximately, $40 \%$ of all proximal DVT will cause PE without treatment [3]. Even with treatment, $1.5 \%$ of patients will have a fatal recurrent PE by 10 years $[4 \bullet]$.

The mainstay of treatment for venous thromboembolism is oral anticoagulation [5]. Using data from the IMS Health National Disease and Therapeutic Index (NDTI) database, one study extrapolated that overall outpatient anticoagulation treatment visits were increasing quarterly from 2009 to 2014 with 2.83 million visits in 2014 [5]. The inferior vena cava filter (IVCF) is an additional tool in the management algorithm of venous thromboembolism (VTE). In the 8-year follow-up of the Prevention $d u$ Risque d'Embolie Pulmonaire par Interruption Cave (PREPIC) study cohort, $35 \%$ of patients in both non-IVCF and IVCF groups had been continued on anticoagulation for the entire study period [6]. Given the clinical overlap between IVCF and anticoagulation, this review will discuss the role of IVCF retrieval in patients on chronic anticoagulation.

\section{Indications for chronic anticoagulation}

Many types of anticoagulation exist, each with its own efficacy and safety profile, including unfractionated heparin, low molecular weight heparin, direct thrombin inhibitors, factor Xa inhibitors, and warfarin [7]. Typical indications for the use of anticoagulation in an ambulatory setting include stroke prevention in patients with atrial fibrillation, atrial flutter, or valvular disease; thrombosis prophylaxis in patients with mechanical heart valves; treatment and secondary prevention of VTE; continuation of prophylaxis for VTE in postsurgical patients; and thromboembolism prevention in specific patient populations such as those with heritable or acquired thrombophilias [7]. For the first episode of DVT or PE, 3 months of anticoagulation is recommended [8]. Indications for extended to indefinite anticoagulation are listed in Table 1 [8, 9]. The use of long-term anticoagulation therapy in the ambulatory setting is increasing, particularly after the introduction of direct oral anticoagulants [5].

\section{Recurrent VTE despite anticoagulation}

Recurrent VTE is a risk factor for death after PE [10]. Rates of recurrent VTE after completing treatment with anticoagulation in patients without cancer or a

\section{Table 1. Indications for extended anticoagulant therapy $[8,9]$}

Unprovoked proximal DVT or PE and low to moderate bleeding risk

Active cancer and VTE

Antiphospholipid antibody syndrome and history of arterial or venous embolism

Atrial fibrillation and high risk of stroke

Status post-stent placement in patients with high risk of stroke

Mechanical heart valves 
known hemophilia have been reported between 11 and 12\% [11, 12]. A recent meta-analysis by Khan et al. reported an incidence of recurrence of $25.2 \%$ and $36.1 \%$ at five and 10 years after the discontinuation of anticoagulation, respectively [4•]. The risk of fatal recurrent VTE at 10 years after the discontinuation of treatment was $1.5 \%[4 \bullet]$. It is possible that VTE recurrence rates are underestimated as many fatal PE are missed [10]. Even despite active anticoagulation therapy, VTE recurrence is estimated to be $2-4 \%$ in 3 months in patients taking warfarin and $4 \%$ in patients on unfractionated heparin or low molecular weight heparin [13]. A randomized, controlled trial comparing dabigatran and warfarin found VTE recurrence rates while on anticoagulation to be $2.4 \%$ and $2.1 \%$, respectively [14]. Predictors of recurrence include male sex (relative risk of 3.6 with 95\% confidence interval of 2.3-5.5), elevated factor VIII (relative risk of 3.4 with 95\% confidence interval of 2.1-5.6), previous symptomatic PE (relative risk of 1.7 with $95 \%$ confidence interval of 1.2-2.5), and increasing patient age (relative risk of 1.2 per 10-year increase in age with 95\% confidence interval of 1.1-1.4) [11]. Patients whose incident VTE was related to surgery have a low incidence of recurrence compared to patients with incident unprovoked VTE who have higher recurrence rates, up to 19.4\% [12]. Patients with a diagnosis of malignancy have over a twofold increased risk of VTE recurrence, and for patients undergoing chemotherapy, the risk is fourfold [10]. Total duration of anticoagulation therapy does not appear to influence the risk of VTE recurrence once therapy has ended [2].

\section{Risk of bleeding on anticoagulation}

Major bleeding while on anticoagulation may have implications for patient care and is associated with morbidity and mortality [15]. A meta-analysis of several randomized controlled trials evaluating patients with atrial fibrillation being treated with anticoagulation versus aspirin found that treatment with anticoagulation increased the rate of major bleeding and $15.3 \%$ of major bleeding events were fatal [16]. The overall rate of major bleeding while on oral anticoagulation was $2.2 \%$ [16]. One study using data from the RIETE registry found a $2.3 \%$ incidence of major bleeding while on anticoagulation for symptomatic acute VTE with a 33\% mortality rate in the first 30 days after the bleeding incident [17]. Additionally, the incidence of recurrent VTE after a major bleeding event was $4.9 \%$ with $1.2 \%$ of patients suffering a fatal PE [17]. A later analysis reported a $2.24 \%$ incidence of major bleeding with a $24.7 \%$ mortality rate (overall fatal bleeding rate of $0.5 \%$ ) [18]. Other studies report major bleeding rates of $1.6-3.2 \%$ while on anticoagulation [14, 19]. The most common sites of major bleeding are gastrointestinal, intracranial, genitourinary, intra-articular, and intramuscular; intracranial bleeding was most commonly fatal $[14,17,18]$. Factors associated with increased risk of fatal bleeding include patient age $>75$ years, body weight $<70 \mathrm{~kg}$, recent major bleeding, chronic heart disease, cancer, and recent immobility for 4 days or more [18].

\section{Rationale for inferior vena cava filtration}

The IVCF has undergone evolution from the original Mobin-Uddin umbrella permanent filter in 1967 to the multitude of optional filters, which may be 
retrieved or left permanently, in use today [3]. The purpose of the IVCF remains to prevent significant PE by physically trapping venous emboli traveling from the lower extremities [20]. The PREPIC study group showed that among patients with proximal DVT who are at high risk for PE, adjunctive placement of a permanent IVCF in addition to anticoagulation therapy, reduced the incidence of asymptomatic or symptomatic PE, albeit with no effect on mortality and an increase in the risk of recurrent DVT [21]. A more recent meta-analysis analyzed data from eleven randomized controlled trials, including the aforementioned PREPIC as well as the PREPIC-II trials, and prospective controlled observational studies of patients at risk for PE who received IVCF compared to those who did not receive an IVCF [22•]. The combined data in this analysis confirmed previous findings that the use of an IVCF reduced the risk of PE, without significantly affecting PE-related or all-cause mortality, and increased the risk of subsequent DVT [22•]. Societal guidelines, such as those by the Society of Interventional Radiology (SIR), the American College of Radiology (ACR), and the American College of Chest Physicians (ACCP), concur that absolute indications for its use include proximal DVT with a contraindication to anticoagulation and proximal DVT in the setting of failed anticoagulation therapy $[3,9,20,23 \bullet]$. These absolute indications are in line with the Food and Drug Administration's (FDA) approved indications for IVCF, which also include emergent treatment for massive PE and chronic, recurrent PE when anticoagulation has failed or is contraindicated [24].

Usage outside of the absolute indications comprises the "relative" indications for IVCF which are controversial with current lack of abundant supporting evidence and include the following: patients without a venous thromboembolism who have endured major trauma, patients at a high risk for VTE such as immobilized patients or intensive care patients, patients with a free-floating iliocaval thrombus, patients with a massive PE and residual DVT regardless of anticoagulation status, and patients with severe cardiopulmonary disease regardless of anticoagulation status [3, 20, 23•]. A study by Angel et al. reported that $58 \%$ of IVCF had been placed for prophylactic use while $42 \%$ had been placed for therapeutic use [25]. Studies report IVCF placement for absolute indications where there is guideline consensus in $62.1-75.9 \%$, for prophylactic purposes in 17.9-27.6\%, and for relative indications in 5.7\% [19, 26•, 27•].

\section{Inferior vena cava filter retrieval}

The FDA has recommended considering retrieval of IVCF as soon as clinically appropriate with an optimal time of retrieval between 29 and 54 days after placement, in order to maximize the benefits of filtration while minimizing the likelihood of complications, although, in practice, the decision to retrieve an IVCF and when is made on a patient-by-patient basis [3]. In order to facilitate this clinical decision-making, the SIR released guidelines for the use of optional IVCF in 2006 stating that an IVCF may be retrieved when an indication for permanent filtration is not present; the risk of clinically significant PE is acceptably low due to a proven ability to sustain appropriate primary therapy or a change in clinical status; the patient is not anticipated to return to a high-risk state for PE due to interruption in primary treatment, change in clinical management, or change in clinical condition; the life expectancy of the patient is long enough to benefit 
from retrieval of the filter; the filter may be safely retrieved or converted; and the patient agrees to have the filter retrieved or converted [20].

Charalel et al. noted that rates of IVCF placement were uptrending each year from 2005 to 2009, with 52 cases per 100,000 in 2009. Beginning in 2010, the same year as the FDA's safety communication regarding IVCF; through 2014, there was a 29\% decrease in the rates of IVCF placement each year, with 36-39.1 cases per 100,000 in 2014 [28 $\bullet$,29]. Additionally, rates of IVCF retrieval increased after 2010; yet, retrieval rates remained low with $3.5 \%$ of filters removed by 1 year follow-up with a mean time to retrieval of 3.4 months and $4.2 \%$ of filters removed by 5 -year follow-up [28•]. Retrospective analyses of nationwide Medicare data similarly showed increasing rates of IVCF retrievals between 2012 and $2016[30 \bullet, 31 \bullet]$. Studies report rates of retrieval ranging from 11.6 to $45 \%$ with a mean time to retrieval of 72 days and a $49 \%$ retrieval attempt rate with a median time to first retrieval attempt of 48 days $[25,26 \bullet, 31 \bullet]$. Several studies cite retrieval success rates ranging from 89 to $96.5 \%[25,26 \bullet, 27 \bullet, 32]$.

\section{Rationale for IVCF retrieval}

As the rates of IVCF placement increased each year in the early 2000s, the reporting of complications related to the device also increased, leading to the FDA safety communications regarding IVCF in 2010 and 2014 [3, 28•]. The most frequently reported complications include IVC thrombosis, IVC perforation, IVCF migration, IVCF fracture, IVCF embolization, IVCF tilt, and recurrent VTE $[3,19,25,28 \bullet, 33]$. Previous studies have shown that complications become more frequent with prolonged IVCF dwell time ( $>30$ days) $[24,25]$. A recent retrospective cohort analysis of an all-payer dataset found that the risk of developing a complication related to an IVCF within one, three, and 5 years was $1.5 \%, 1.7 \%$, and $1.8 \%$, respectively, and that complications were more likely to occur within the first 3 months $(85.2 \%$ of complications reported within 3 months) [28•]. The most common complication in this study was IVC thrombosis, which comprised $90 \%$ of the complications by 5 years [28•]. Similarly, another study reported a $17.7 \%$ complication rate within a mean of 32 days with the most frequent complications being DVT, PE, and IVC thrombosis and an adverse clinical outcome rate of $42.7 \%$, which included those patients who died, had recurrent VTE, or IVC thrombosis [19]. These investigators found that IVCF migration, severe tilting, embolization, fracture, infection, and IVC stenosis were much less common complications (0.1-0.4\%) [19]. Another study noted a complication rate of $22.2 \%$, with IVC thrombosis the most common complication and most complications documented in the first 28 days after insertion [33]. Younger patients and those on anticoagulation are less likely to have complications related to an indwelling IVCF [19]. Differences in reported complication rates among various studies likely reflect differences in study design and definition of complications, including the classification of what is significant or major.

\section{Technical aspects of retrieval}

Routine IVCF retrieval, also known as standard, "snare and sheath," or "snareassisted" retrieval, refers to the use of a snare to engage the hook of the filter with 
subsequent collapsing of the filter into a sheath (Fig. 1) [3, 32]. Routine retrieval techniques are used in $79-90.2 \%$ of retrieval procedures and have reported success rates of $73.2-100 \%$ [27•, 32, 34-36]. Advanced techniques for more challenging IVCF retrievals, when the routine retrieval is unsuccessful, include maneuvers such as the loop-snare technique, the Hangman technique (Fig. 2), endobronchial forceps-assisted technique (Fig. 3), balloon displacement, and the Excimer laser sheath-assisted technique [3, 36-39]. Common reasons for routine retrieval failure with the subsequent need for advanced retrieval techniques include severe tilt, migration, penetration into the caval wall, caval occlusion (Fig. 4), and hook embedment into the IVC wall [3, 32, 34, 36, 37, 40-42]. Longer filter dwell time has been associated with the need for advanced techniques for retrieval $[35,41]$. Advanced techniques have reported success rates of $62.9-100 \%$ and lead to higher IVCF retrieval rates, up to $98.2 \%$ [27•, $36,37,40,43 \bullet]$. They are associated with longer fluoroscopy times and greater financial burden $[27 \bullet, 32]$.

\section{Complications of retrieval}

Complications are reported in $1-7.9 \%$ of IVCF retrieval procedures, with increased rates of complications reported with advanced techniques compared to routine retrievals $[27 \bullet, 34,35]$. Importantly, the use of anticoagulation has not been found to be associated with filter retrieval complications [27•]. One study noted major complications, such as IVC tear, hematoma, pneumothorax, or surgical retrieval, in $2.8 \%$ of retrievals and minor complications in $1.2 \%$ [19]. Other reported complications include IVC intussusception, IVC stenosis, failure of filter collapse into the sheath, strut fractures with migration, and IVC
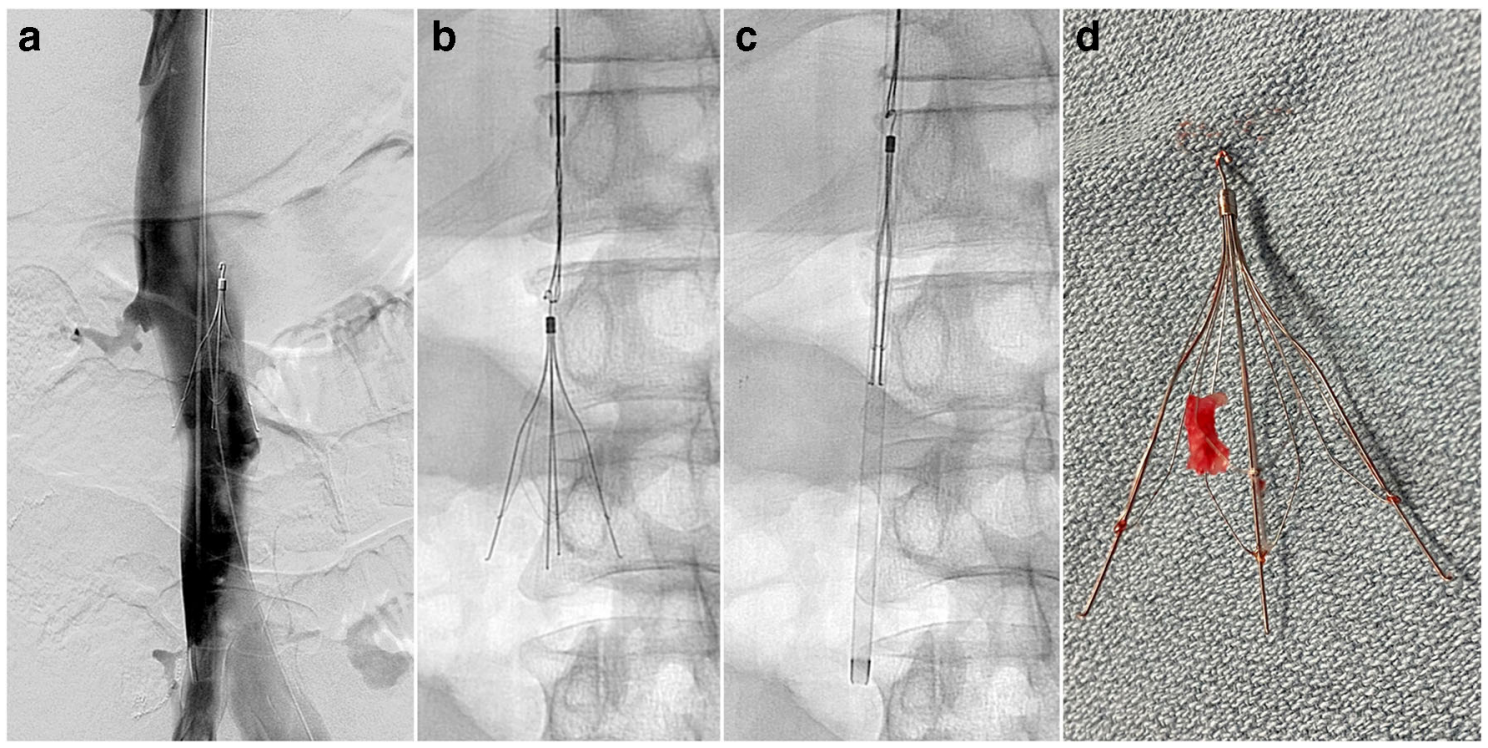

Fig. 1. Snared-assisted filter retrieval. a Inferior vena cava venography demonstrating a patent inferior vena cava without caval thrombosis. A tip-centered Gunther Tulip inferior vena cava filter was visualized. $\mathbf{b}$ A snare was advanced around the apex of the inferior vena cava filter and secured. $\mathbf{c}$ A sheath was then advanced over the inferior vena cava filter and the filter was removed. $\mathbf{d}$ Gross pathologic image of the intact Gunther Tulip inferior vena cava filter. 

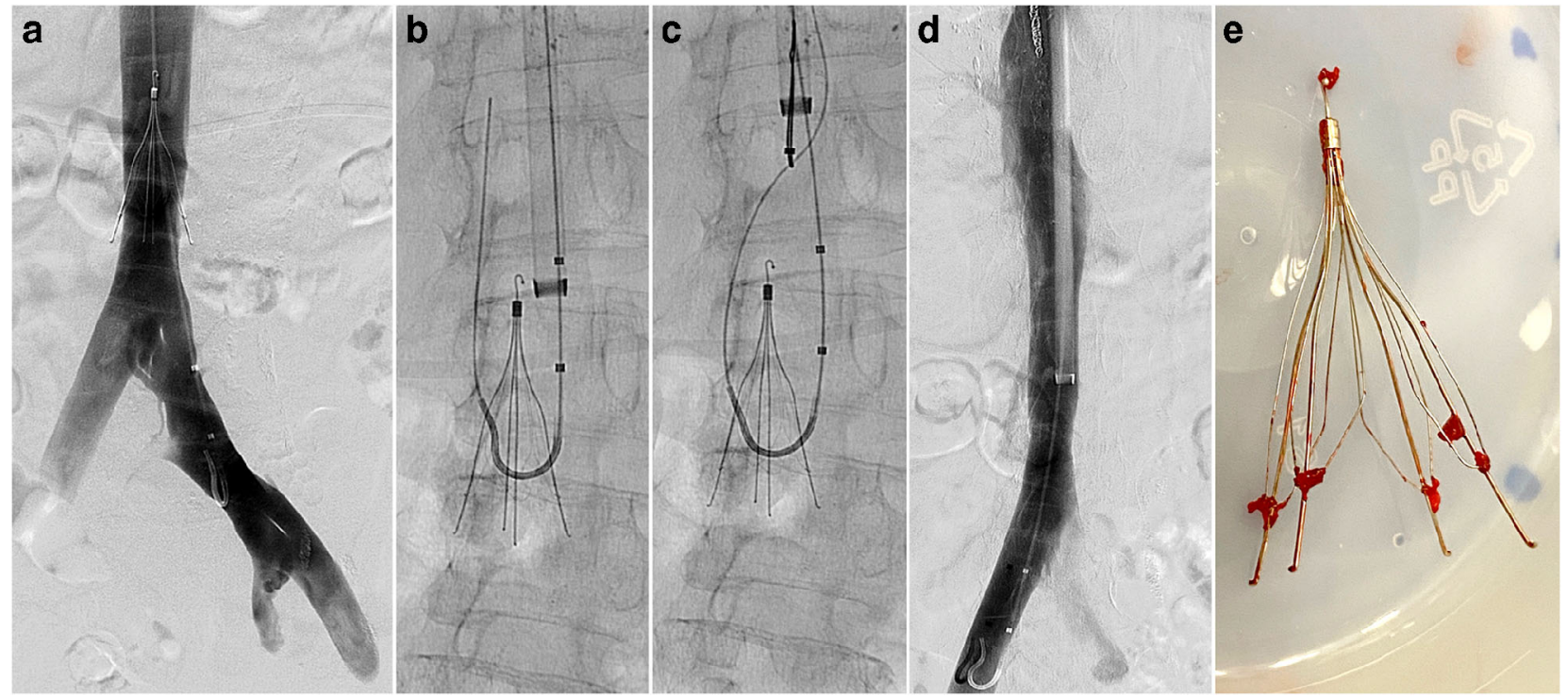

Fig. 2. Hangman-assisted filter retrieval. a Inferior vena cava venography showing a patent inferior vena cava without caval thrombosis. A tip-centered Gunther Tulip inferior vena cava filter was visualized. b An Omni flush catheter was advanced under the apex of the inferior vena cava filter and a hydrophilic wire was advanced. $\mathbf{c}$ The hydrophilic wire was captured with a snare to create the hangman. The Omni flush catheter was subsequently removed. The sheath was then advanced over the inferior vena cava filter and the filter was removed. $\mathbf{d}$ Gross pathologic image of the intact Gunther Tulip inferior vena cava filter.

dissection $[27 \bullet, 35,43 \bullet]$. A recent study noted a trend toward the occurrence of hematomas more frequently in patients who underwent endobronchial forceps retrievals $[43 \bullet]$.

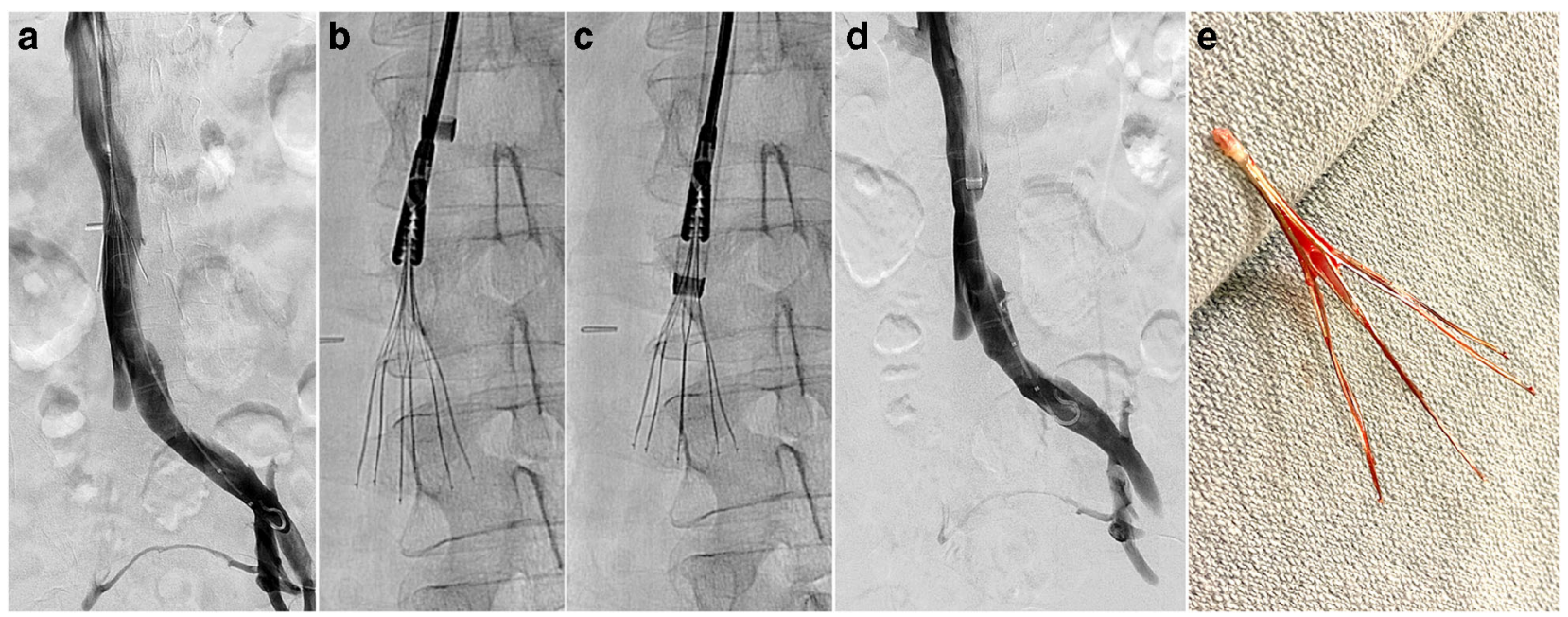

Fig. 3. Endobronchial forceps-assisted filter retrieval. a Inferior vena cava venography demonstrating a patent inferior vena cava without caval thrombosis. An Argon Option filter was visualized. $\mathbf{b}$ The endobronchial forceps were used to grasp the apex of the inferior vena cava filter. $\mathbf{c}$ The sheath was then advanced over the inferior vena cava filter and the filter was removed. $\mathbf{d}$ Postretrieval inferior vena cava venography showing no caval transection, intussusception, spasm, or thrombosis. e Gross pathologic image of the intact Argon Option inferior vena cava filter. 


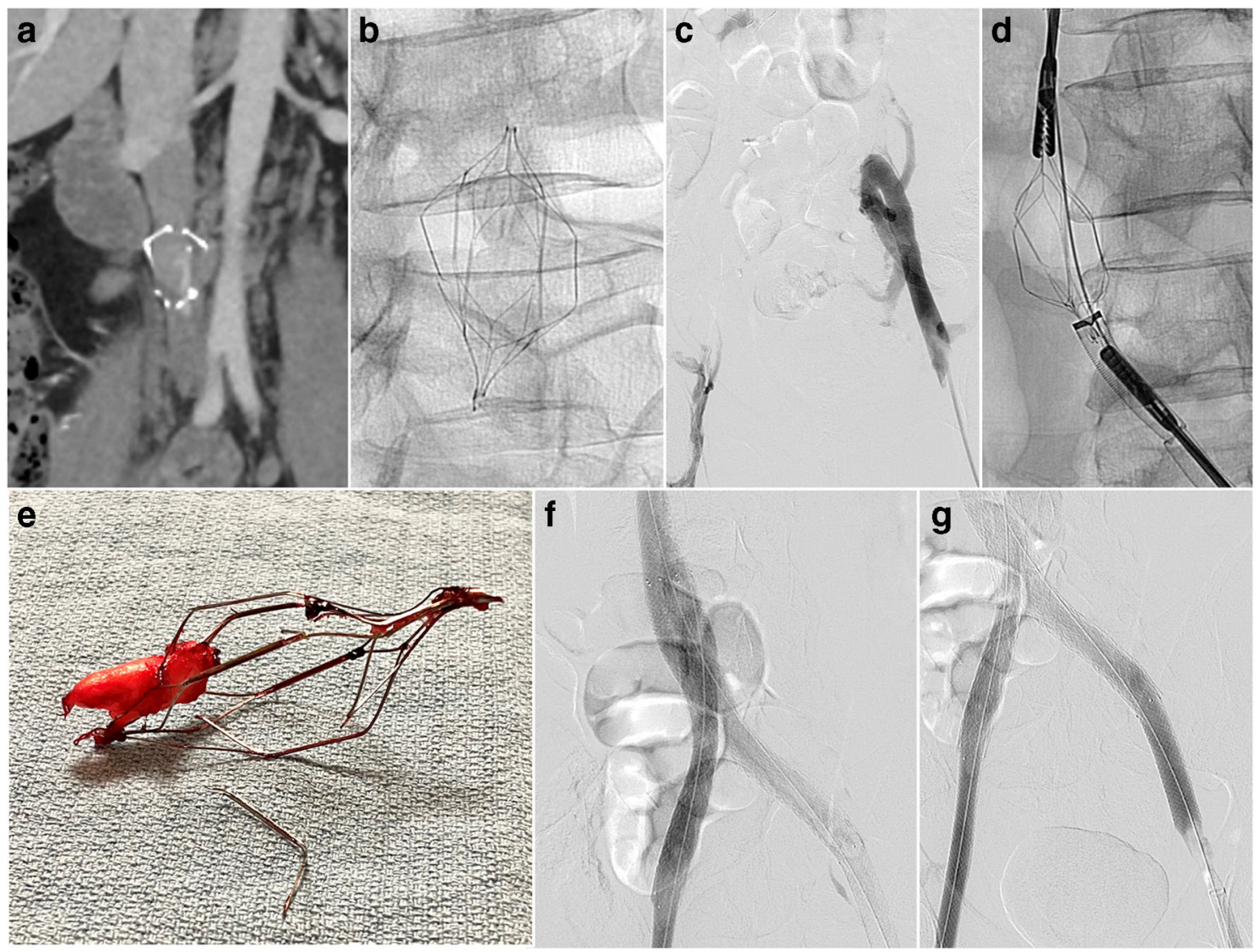

Fig. 4. TrapEase inferior vena cava filter-associated iliocaval thrombosis. a Coronal computed tomographic image with contrast showing thrombosis of the inferior vena cava from a TrapEase inferior vena cava filter. $\mathbf{b}$ Spot magnification radiograph demonstrating the fractured TrapEase inferior vena cava filter. c Bilateral ascending iliocaval venography showing chronic occlusion of both iliocaval venous segments from the TrapEase inferior vena cava filter. $\mathbf{d}$ The filter was grasped at both apices using endobronchial forceps and removed through the left groin sheath. $\mathbf{e}$ Gross pathologic image of the fractured TrapEase inferior vena cava filter. Completion bilateral ascending iliocaval venography of the $(\mathbf{f})$ upper and $(\mathbf{g})$ lower venous stent reconstruction demonstrating brisk inline flow from both common femoral veins to the inferior vena cava.

\section{IVCF retrieval in patients on anticoagulation}

Given the known potential for complications of indwelling IVCF, retrieval of those deemed no longer clinically necessary is warranted. As previously discussed, the rate of IVCF retrieval has increased since the FDA communications in 2010 and 2014, but overall remains low [28•, 30•]. Even in filters placed with the intention of retrieval at a later date, approximately, $40 \%$ are made permanent, due to various reasons such as persistent indication, death, and loss to follow-up [44]. In one retrospective study, anticoagulation was restarted in $72.4 \%$ of patients and appropriately dosed in $66 \%$ within 30 days of IVCF placement; yet, the IVCF retrieval attempt rate was $43.4 \%$, excluding 
patients who had died or developed permanent indications for filtration [19]. The same study noted that the time to attempted retrieval was longer than the time to anticoagulation regardless of the indication for IVCF placement with a median time from anticoagulation to IVCF retrieval of 81 days [19]. Factors that have been associated with greater likelihood of IVCF retrieval are younger patient age and more dedicated follow-up visits [19]. Angel et al. found that the most common reasons for not removing a retrievable IVCF were loss to follow-up and continued risk of PE with or without the ability to initiate anticoagulation [25]. Once the decision to retrieve the IVCF is made, prophylactic or therapeutic anticoagulation need not be reversed or held for the retrieval procedure as there is no increased risk of hemorrhage [45]. Determination of timing for retrieval is made on a patient-by-patient basis with consideration of a multitude of patient factors [3]. The following sections include discussion regarding the role of IVCF retrieval in specific patient populations likely to be concurrently on chronic anticoagulation therapy.

\section{Retrieval in patients with a previous contraindication to anticoagulation}

Optional filters that are later converted to permanent were commonly placed initially due to a contraindication to anticoagulation, presumably due to a persistent contraindication [44]. Only a fraction of those patients who are restarted on their anticoagulation after resolution of the contraindication have their filters retrieved and there is substantial lag time between the resumption of anticoagulation and the IVCF retrieval [19]. Approximately, $62.1 \%$ of patients who had a contraindication to anticoagulation due to reasons such as acute bleeding or surgery and had an IVCF placed due to known VTE were restarted on appropriately dosed anticoagulation within 5.5 days of IVCF placement [19]. It stands to reason that this fraction of patients should undergo evaluation to have their optional IVCF removed shortly thereafter. Furthermore, identification of patients within this population who may not ultimately resolve their contraindication to anticoagulation and consideration for placement of a permanent filter rather than an optional filter could be beneficial.

\section{Retrieval in patients with filter thrombus}

Due to the nature of the device, not infrequently thrombus is discovered in the IVCF at the time of retrieval. Filter thrombus may range from small asymptomatic thrombus discovered incidentally to complete IVC occlusion with associated clinical symptoms [46]. IVCF thrombus was encountered in up to $9 \%$ of retrievals in one study [41]. Retrieval was postponed in these cases and approximately 53\% of these IVCF were later removed [41]. The remaining $47 \%$ of these patients were lost to follow-up and their filters were not retrieved [41]. Anticoagulation should be started or maintained in these patients with any amount of filter thrombus and no contraindication to anticoagulation; however, the efficacy of anticoagulation in facilitating the resolution of the filter thrombus is not clear $[46,47]$. Filter thrombus of any extent in a patient without a 
previously documented VTE constitutes a new diagnosis of VTE and primary therapy should be started or maintained and the filter should not be retrieved [20]. Filter retrieval should be reconsidered at a later date [20]. Certain patients may benefit from more extensive endovascular therapies, such as catheter-directed thrombolysis, mechanical thrombectomy, venoplasty, and stent placement (Fig. 5) [46]. There is currently limited published data and no current specific guidelines to support decision-making in the situation of filter thrombus in patients with previously diagnosed VTE, although the SIR Consensus Guidelines
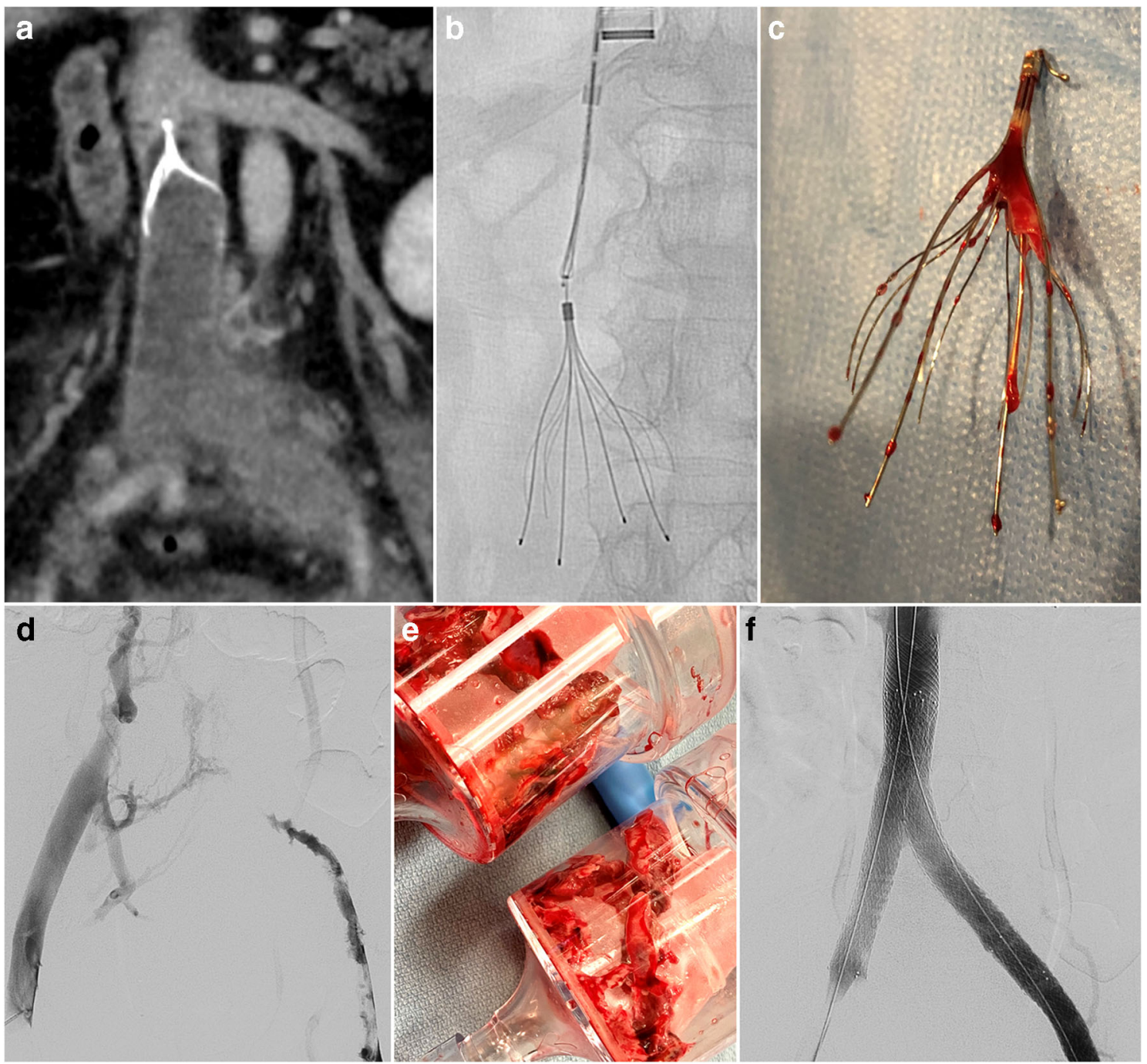

Fig. 5. Celect inferior vena cava filter-associated iliocaval thrombosis. a Coronal computed tomographic image with contrast showing thrombosis of the inferior vena cava from a Celect inferior vena cava filter. $\mathbf{b}$ The Celect inferior vena cava filter was retrieved using a snare. $\mathbf{c}$ Gross pathologic image of the Celect inferior vena cava filter. $\mathbf{d}$ After retrieval, bilateral ascending iliocaval venography showed acute-on-chronic occlusion of both iliocaval venous segments. e AngioVac suction thrombectomy of the bilateral iliocaval segments was performed. $\mathbf{f}$ Completion bilateral ascending iliocaval venography, after stent reconstruction, demonstrating brisk inline flow from both common femoral veins to the inferior vena cava. 
recommend an individualized clinical assessment of the risk of PE at the time of retrieval given the thrombus burden and management based on the clinical judgment of the physician [20].

\section{Retrieval in patients on anticoagulation due to the presence of} IVCF

The ACCP and American Heart Association (AHA) recommend against anticoagulation solely due to the presence of an IVCF $[9,19,48]$. Findings from a recent study support this guideline due to the risk of bleeding on anticoagulation balanced against a relatively narrow window of potential benefit of protection from IVCF-related complications [19]. Furthermore, anticoagulation has been shown to have limited efficacy in the management and prevention of filter thrombus and IVC occlusion [47]. The patient should be re-evaluated to determine if the clinical need for the IVCF remains. If no clinical indication for the IVCF remains, the IVCF should be retrieved if deemed safe to do so. If the patient still requires the IVCF after re-evaluation or the IVCF is unable to be retrieved, the IVCF should remain but the patient need not be maintained on anticoagulation if another indication does not exist.

\section{Retrieval in patients on anticoagulation for unprovoked DVT}

Patients with a first episode of unprovoked DVT or PE are managed first-line with anticoagulation therapy for at least 3 months [9]. A second episode of unprovoked VTE warrants extended anticoagulation therapy in those with lowmoderate risk of bleeding [9]. Those with a second unprovoked VTE and high risk of bleeding should be managed with at least 3 months of anticoagulation and evaluation of the risks and benefits of anticoagulation should be done thereafter [9]. There is no specific published data indicating that there is any difference in IVCF efficacy or safety in this patient population. Thus, retrieval of IVCF in this population should be regarded with the same clinical judgment as that used in other patients and consideration for retrieval should be made once there is no longer an indication for filtration and given retrieval is deemed safe.

\section{Retrieval in patients with hypercoagulable disorders}

Patients with hypercoagulable disorders, such as antiphospholipid syndrome (APS), are managed on extended anticoagulation therapy and the use of an IVCF in this subset of patients is controversial $[9,49 \bullet]$. Previous reports of recurrent PE with thrombus proximal and distal to a permanent IVCF in patients with APS suggested that permanent IVCF placed in this population could be more problematic than beneficial [50]. A small case series evaluated the use of optional IVCF in patients with APS who required temporary protection from PE while they had a contraindication to anticoagulation due to surgery or major bleeding or when they were suffering ongoing thrombosis despite anticoagulation therapy [49•]. At the time of filter retrieval in these patients (within 2 months of placement), none had suffered a PE and there was no thrombus noted on the retrieved filters [49•]. This study suggests that 


\section{Impact of filter retrieval}

optional IVCF may be safe to use in this population for a short period of time while additional protection from PE is required or a temporary contraindication to anticoagulation exists [49•]. Even in this small case series, one patient was lost to follow-up and the filter was never retrieved, highlighting the risk that despite the best intentions, many filters will not be removed and pose particular risk in this subset of patients in the long term [49•]. An additional consideration in this population is that patients with hypercoagulable disorders have been found to be more likely to require an advanced retrieval [27•]. Certainly, this population of patients requires stringent patient selection and close follow-up and monitoring until their IVCF may be safely retrieved.

Besides prevention of the aforementioned potential complications of indwelling IVCF, IVCF retrieval has also recently been associated with decreased incidence of post-thrombotic syndrome (PTS) [43•]. Previous studies have shown no relationship between IVCF and the long-term development of PTS; still, the increased risk of lower extremity DVT in patients with indwelling IVCF is well established [6].

Another factor to consider is the risk of recurrent DVT and PE after IVCF retrieval. A recent study by Brahmandam et al. found that recurrent VTE occurred in 3.6\% of patients on follow-up after IVCF retrieval [27 •]. Another study found that $26 \%$ of patients with incident DVT without PE had a PE after filter retrieval [28•]. The same study found that the risk of PE was highest in the first 3 months after IVCF placement [28•]. Consideration of each individual patient's risk for recurrent VTE is vital before making the decision to retrieve the IVCF.

\section{Factors that improve rates of filter retrieval}

Due to persistently low rates of IVCF retrieval, efforts to increase rates of retrieval are warranted. Strategies including an IVCF database with alert mechanism, automated email reminder system, dedicated IVCF clinics, dedicated IVCF staff to coordinate patient follow-up, inclusion of IVCF follow-up in hospital discharge planning, semi-automated filter tracking application, and more patienttargeted efforts such as increased patient education, phone calls, and letters have been described with varying rates of success [51-54]. Dedicated follow-up is consistently the most influential factor for increasing rates of IVCF retrieval [19]. Several studies emphasize the importance of shifting responsibility of IVCF management and follow-up from the referring physician to the physician who placed the filter [51].

\section{Future perspectives}

Ongoing developments to combat low IVCF retrieval rates include options such as bioabsorbable or bioconvertible filters which obviate the need for retrieval and obligate temporary filters which necessitate removal [3]. The SENTRY bioconvertible filter design involves a nitinol cylindrical 
frame with bioabsorbable filament that holds the filter arms in the central portion of the IVC lumen, and when the filament degrades, the filter arms are released so that the IVC is left patent and no longer filtered [55•]. Recent results from the first year of analysis of the SENTRY filter in a prospective nonrandomized trial show promising rates of technical and clinical success in patients requiring temporary protection from PE [55•]. A completely absorbable filter design made from polydioxanone is being investigated in swine and appears to be effective in trapping thrombi [56]. The Angel Catheter is a triple lumen central venous catheter with an integrated nitinol conical filter designed for the purpose of obligate temporary $(<30$ days $)$ IVC filtration in critically ill patients who are at high risk of PE [57]. The device may be placed at the patient's bedside without the use of fluoroscopy and has recently been approved by the FDA for use in North America [57]. Multiple studies have shown that the Angel Catheter is effective at filtering thrombus and preventing PE, but safety concerns including inadvertent device removal, infection risk, and device migration remain [57].

\section{Conclusion}

The rates of IVCF retrieval have been increasing since the FDA safety communications in 2010 and 2014; however, they remain low. Previous studies have shown that longer filter dwell times are associated with greater risk for indwelling filter complications and more difficulty with filter retrieval, although recent studies suggest that complications are more frequent in the first 30 days after placement. These findings suggest that while filters should be removed as early as is clinically safe, if a clinical indication for the optional filter remains or retrieval is not deemed safe or possible, conversion to a permanent filter is a reasonable option. Advanced retrieval techniques in the hands of experienced interventionalists enable increased retrieval success even in the most challenging of cases; however, these techniques are associated with greater risk of retrieval complications and higher cost to the healthcare system. The decision to retrieve a filter is ultimately made on a patient-by-patient basis and requires diligent follow-up with consistent re-evaluation of the clinical need for the indwelling IVCF, particularly in those with concurrent long-term anticoagulation therapy.

\section{Compliance with Ethical Standards}

\section{Conflict of interest}

Jeffrey Forris Beecham Chick is a consultant and speaker for Guerbet and C. R. Bard.

Savannah Fletcher, Adam Plotnik, Ravi N. Srinivasa, and John M Moriarty declare that they have no conflict of interest.

\section{Human and animal rights}

This article does not contain any studies with human or animal subjects performed by any of the authors. 


\section{Open Access}

This article is licensed under a Creative Commons Attribution 4.0 International License, which permits use, sharing, adaptation, distribution and reproduction in any medium or format, as long as you give appropriate credit to the original author(s) and the source, provide a link to the Creative Commons licence, and indicate if changes were made. The images or other third party material in this article are included in the article's Creative Commons licence, unless indicated otherwise in a credit line to the material. If material is not included in the article's Creative Commons licence and your intended use is not permitted by statutory regulation or exceeds the permitted use, you will need to obtain permission directly from the copyright holder. To view a copy of this licence, visit http://creativecommons.org/licenses/by/4.0/.

\section{References and Recommended Reading}

Papers of particular interest, published recently, have been highlighted as:

- Of importance

1. Rosendaal FR. Causes of venous thrombosis. Thromb J. 2016;14:24. https://doi.org/10.1186/s12959-0160108-y.

2. Heit JA. The epidemiology of venous thromboembolism in the community. Arterioscler Thromb Vasc Biol 2008;28:370-2. https://doi.org/10.1161/ATVBAHA. 108.162545

3. Moriarty J, Steinberger J, Bansal A. Inferior vena cava filters: when to place and when to remove. Semin Respir Crit Care Med. 2017;38:084-93. https://doi. org/10.1055/s-0036-1597558.

4. Khan F, Rahman A, Carrier M, Kearon C, Weitz JI, Schulman S, et al. Long term risk of symptomatic recurrent venous thromboembolism after discontinuation of anticoagulant treatment for first unprovoked venous thromboembolism event: systematic review and meta-analysis. BMJ. 2019:14363. https://doi.org/ 10.1136/bmj.l4363

This recent study included 18 randomized controlled trials and prospective cohort studies which included 7515 patients in order to determine rates of recurrent VTE after the discontinuation of anticoagulation. The large sample size and strong study design of the studies included are obvious strengths of this reference. Additionally, the more recent data is likely to better reflect current practice patterns.

5. Barnes GD, Lucas E, Alexander GC, Goldberger ZD. National trends in ambulatory oral anticoagulant use. Am J Med. 2015;128:1300-1305.e2. https://doi.org/ 10.1016/j.amjmed.2015.05.044

6. The PREPIC Study Group. Eight-year follow-up of patients with permanent vena cava filters in the prevention of pulmonary embolism: the PREPIC (Prévention du Risque d'Embolie Pulmonaire par interruption cave) randomized study. Circulation. 2005;112:41622. https://doi.org/10.1161/CIRCULATIONAHA.104. 512834.

7. DeWald TA, Washam JB, Becker RC. Anticoagulants. Neurosurg Clin N Am. 2018;29:503-15. https://doi. org/10.1016/j.nec.2018.06.003.
8. Wigle $\mathrm{P}$, Hein B, Bloomfield HE, Tubb M, Doherty M. Updated guidelines on outpatient anticoagulation. Am Fam Physician. 2013;87:556-66.

9. Kearon C, Akl EA, Ornelas J, Blaivas A, Jimenez D, Bounameaux $\mathrm{H}$, et al. Antithrombotic therapy for VTE disease. Chest. 2016;149:315-52. https://doi.org/10. 1016/j.chest.2015.11.026.

10. Heit JA, Mohr DN, Silverstein MD, Petterson TM, O'Fallon WM, Melton LJ. Predictors of recurrence after deep vein thrombosis and pulmonary embolism: a population-based cohort study. Arch Intern Med. 2000;160:761-8. https://doi.org/10.1001/archinte. 160.6.761.

11. Kyrle PA, Minar E, Bialonczyk C, Hirschl M, Weltermann A, Eichinger S. The risk of recurrent venous thromboembolism in men and women. N Engl J Med. 2004;350:2558-63. https://doi.org/10.1056/ NEJMoa032959.

12. Baglin $\mathrm{T}$, Luddington $\mathrm{R}$, Brown $\mathrm{K}$, Baglin $\mathrm{C}$. Incidence of recurrent venous thromboembolism in relation to clinical and thrombophilic risk factors: prospective cohort study. Lancet. 2003;362:523-6. https://doi.org/ 10.1016/S0140-6736(03)14111-6.

13. Kazmi RS, Lwaleed BA. New anticoagulants: how to deal with treatment failure and bleeding complications: new anticoagulants: dealing with treatment failures and bleeding. Br J Clin Pharmacol. 2011;72:593-603. https://doi.org/10.1111/j.1365-2125.2011.04060.x.

14. Schulman S, Kearon C, Kakkar AK, Mismetti P, Schellong S, Eriksson $\mathrm{H}$, et al. Dabigatran versus warfarin in the treatment of acute venous thromboembolism. N Engl J Med. 2009;361:2342-52. https://doi. org/10.1056/NEJMoa0906598.

15. Rubboli A, Becattini C, Verheugt FW. Incidence, clinical impact and risk of bleeding during oral anticoagulation therapy. World J Cardiol. 2011;3:3518. https://doi.org/10.4330/wjc.v3.i11.351.

16. van Walraven $C$, Hart RG, Singer DE, Laupacis A, Connolly S, Petersen $\mathrm{P}$, et al. Oral anticoagulants vs 
aspirin in nonvalvular atrial fibrillation: an individual patient meta-analysis. JAMA. 2002;288:2441-8. https://doi.org/10.1001/jama.288.19.2441.

17. Nieto JA, Camara T, Gonzalez-Higueras E, RuizGimenez N, Guijarro R, Marchena PJ, et al. Clinical outcome of patients with major bleeding after venous thromboembolism. Findings from the RIETE registry. Thromb Haemost. 2008;100:789-96.

18. Nieto JA, Solano R, Ruiz-Ribó MD, Ruiz-Gimenez N, Prandoni P, Kearon C, et al. Fatal bleeding in patients receiving anticoagulant therapy for venous thromboembolism: findings from the RIETE registry. J Thromb Haemost. 2010;8:1216-22. https://doi.org/10.1111/j. 1538-7836.2010.03852.x.

19. Weinberg I, Abtahian F, DeBiasi R, Cefalo P, MacKay C, Hawkins BM, et al. Effect of delayed inferior vena cava filter retrieval after early initiation of anticoagulation. Am J Cardiol. 2014;113:389-94. https://doi.org/10. 1016/j.amjcard.2013.08.053.

20. Kaufman JA, Kinney TB, Streiff MB, Sing RF, Proctor $\mathrm{MC}$, Becker D, et al. Guidelines for the use of retrievable and convertible vena cava filters: report from the Society of Interventional Radiology Multidisciplinary Consensus Conference. J Vasc Interv Radiol. 2006;17:449-59. https://doi.org/10.1097/01.RVI. 0000203418-39769.0D

21. Decousus H, Leizorovicz A, Parent F, Page Y, Tardy B, Girard P, et al. A clinical trial of vena caval filters in the prevention of pulmonary embolism in patients with proximal deep-vein thrombosis. $\mathrm{N}$ Engl J Med. 1998;338:409-16. https://doi.org/10.1056/ NEJM199802123380701.

22.• Bikdeli B, Chatterjee S, Desai NR, Kirtane AJ, Desai MM, Bracken $\mathrm{MB}$, et al. Inferior vena cava filters to prevent pulmonary embolism. J Am Coll Cardiol. 2017;70:158797. https://doi.org/10.1016/j.jacc.2017.07.775

This recent study combines data from eleven randomized controlled trials and prospective observational cohort studies.

23. Minocha J, Smith AM, Kapoor BS, Fidelman N, Cain TR, Caplin DM, et al. ACR appropriateness criteria ${ }^{\circledR}$ radiologic management of venous thromboembolism-inferior vena cava filters. J Am Coll Radiol. 2019;16:S214-26. https:// doi.org/10.1016/j.jacr.2019.02.010

This reference was included in order to present the most updated guidelines.

24. Morales JP, Li X, Irony TZ, Ibrahim NG, Moynahan M, Cavanaugh KJ. Decision analysis of retrievable inferior vena cava filters in patients without pulmonary embolism. J Vasc Surg Venous Lymphat Disord. 2013;1:376-84. https://doi.org/10.1016/j.jvsv.2013. 04.005 .

25. Angel LF, Tapson V, Galgon RE, Restrepo MI, Kaufman J. Systematic review of the use of retrievable inferior vena cava filters. J Vasc Interv Radiol. 2011;22:15221530.e3. https://doi.org/10.1016/j.jvir.2011.08.024.

26. Hamidizadeh R, Liu D, Khosa F, Chung J, Klass D, Homayoon B. Retrievable inferior vena cava filters: a tertiary centre perspective and quality assurance study. Can Assoc Radiol J. 2019;70:193-8. https://doi.org/10. 1016/j.carj.2018.08.004

This recent study includes data from one institution regarding IVCF retrievals.

27. $\quad$ Brahmandam A, Skrip L, Mojibian H, Aruny J, Sumpio B, Dardik A, et al. Costs and complications of endovascular inferior vena cava filter retrieval. J Vasc Surg Venous Lymphat Disord. 2019;7:653-659.e1. https://doi.org/10.1016/j.jvsv.2019.02.017

This recent study includes data regarding IVCF retrievals as well as cost analysis of standard and advanced retrievals.

28. Charalel RA, Durack JC, Mao J, Ross JS, Meltzer AJ, Sedrakyan A. Statewide inferior vena cava filter placement, complications, and retrievals. Med Care. 2018;56:260-5

This recent study includes analysis of a large sample size of patients with IVCFs in the state of NY using an all-payer dataset to determine overall trends.

29. Reddy S, Lakhter V, Zack CJ, Zhao H, Chatterjee S, Bashir R. Association between contemporary trends in inferior vena cava filter placement and the 2010 Food and Drug Administration advisory. JAMA Intern Med. 2017;177:1373-4. https://doi.org/10.1001/ jamainternmed.2017.2719.

30. Guez D, Hansberry DR, Eschelman DJ, Gonsalves CF, Parker L, Rao VM, et al. Inferior vena cava filter placement and retrieval rates among radiologists and nonradiologists. J Vasc Interv Radiol. 2018;29:482-5. https://doi.org/10.1016/j.jvir.2017.11.008

This recent study used data from a nationwide Medicare dataset in order to assess overall trends of IVCF placement and retrieval rates. The large and nationwide nature of the patient sample is a strength of this study; however, the data is limited to patients with Medicare coverage.

31. Ahmed O, Wadhwa V, Patel K, Patel MV, Turba UC, Arslan B. Rising retrieval rates of inferior vena cava filters in the United States: insights from the 2012 to 2016 summary Medicare claims data. J Am Coll Radiol. 2018;15:1553-7. https://doi.org/10.1016/j. jacr.2018.01.037

This recent study also used data from a Medicare dataset in order to assess overall trends in IVCF placement and retrieval. This study has similar strengths and weaknesses to the similar above study.

32. Dowell JD, Wagner D, Elliott E, Yildiz VO, Pan X. Factors associated with advanced inferior vena cava filter removals: a single-center retrospective study of 203 patients over 7 years. Cardiovasc Intervent Radiol. 2016;39:218-26. https://doi.org/10.1007/s00270015-1256-3.

33. Sarosiek S, Crowther M, Sloan JM. Indications, complications, and management of inferior vena cava filters: the experience in 952 patients at an academic hospital with a level I trauma center. JAMA Intern Med. 2013;173:513-7. https://doi.org/10.1001/ jamainternmed.2013.343.

34. Neill M, Charles HW, Pflager D, Deipolyi AR. Factors associated with reduced radiation exposure, cost, and technical difficulty of inferior vena cava filter 
placement and retrieval. Proc (Baylor Univ Med Cent). 2017;30:21-5.

35. Al-Hakim R, Kee ST, Olinger K, Lee EW, Moriarty JM, McWilliams JP. Inferior vena cava filter retrieval: effectiveness and complications of routine and advanced techniques. J Vasc Interv Radiol. 2014;25:933-9. https://doi.org/10.1016/j.jvir.2014.01.019.

36. Lynch FC. Modified loop snare technique for the removal of bard recovery, G2, G2 express, and eclipse inferior vena cava filters. J Vasc Interv Radiol. 2012;23:687-90. https://doi.org/10.1016/j.jvir.2012. 01.060 .

37. Al-Hakim R, McWilliams JP, Derry W, Kee ST. The hangman technique: a modified loop snare technique for the retrieval of inferior vena cava filters with embedded hooks. J Vasc Interv Radiol. 2015;26:107-10. https://doi.org/10.1016/j.jvir.2014.04.002.

38. Vink TWF, Reekers JA, Van Dijk LC, Wever JJ, Van Overhagen H. Balloon-assisted retrieval of tilted OptEase IVC filter. Cardiovasc Intervent Radiol. 2012;35:975-7. https://doi.org/10.1007/s00270-011-0332-6.

39. Kuo WT, Odegaard JI, Louie JD, Sze DY, Unver K, Kothary N, et al. Photothermal ablation with the excimer laser sheath technique for embedded inferior vena cava filter removal: initial results from a prospective study. J Vasc Interv Radiol. 2011;22:813-23. https://doi.org/10.1016/j.jvir.2011.01.459.

40. Stavropoulos SW, Ge BH, Mondschein JI, ShlanskyGoldberg RD, Sudheendra D, Trerotola SO. Retrieval of tip-embedded inferior vena cava filters by using the endobronchial forceps technique: experience at a single institution. Radiology. 2015;275:900-7. https://doi. org/10.1148/radiol.14141420.

41. Gotra A, Doucet C, Delli Fraine P, Bessissow A, Dey C, Gallix B, et al. Predicting inferior vena cava (IVC) filter retrievability using positional parameters: a comparative study of various filter types. Diagn Interv Imaging. 2018;99:615-24. https://doi.org/10.1016/j.diii.2018. 04.003 .

42. Bae JH, Lee SY. Filter tilting and retrievability of the Celect and Denali inferior vena cava filters using propensity score-matching analysis. Eur J Radiol Open. 2018;5:153-8. https://doi.org/10.1016/j.ejro.2018.09. 001.

43. Chen S, Gao Y, Wang W, Yu C, Tang W, Wang X, et al. Long-term clinical outcomes of complicated retrievable inferior vena cava filter for deep venous thrombosis patients: safety and effectiveness. Med Sci Monit. 2019;25:128-34. https://doi.org/10.12659/MSM. 911813

This recent study followed a cohort of 79 patients who had undergone IVCF retrieval for up to 24 months in order to assess long-term outcomes.

44. Yoon DY, Vavra AK, Eifler AC, Teter K, Eskandari MK, Ryu RK, et al. Why temporary filters are not removed: clinical predictors in 1,000 consecutive cases. Ann Vasc Surg. 2017;42:64-70. https://doi.org/10.1016/j.avsg. 2016.10.055.
45. Hoppe H, Kaufman JA, Barton RE, Petersen BD, Lakin PC, DeLoughery TG, et al. Safety of inferior vena cava filter retrieval in anticoagulated patients. Chest. 2007;132:31-6. https://doi.org/10.1378/chest.062897.

46. Sildiroglu O, Ozer H, Turba UC. Management of the thrombosed filter-bearing inferior vena cava. Semin Interv Radiol. 2012;29:57-63. https://doi.org/10. 1055/s-0032-1302453.

47. Ahmad I, Yeddula K, Wicky S, Kalva SP. Clinical sequelae of thrombus in an inferior vena cava filter. Cardiovasc Intervent Radiol. 2010;33:285-9. https:// doi.org/10.1007/s00270-009-9664-X.

48. Jaff MR, McMurtry MS, Archer SL, Cushman M, Goldenberg N, Goldhaber SZ, et al. Management of massive and submassive pulmonary embolism, iliofemoral deep vein thrombosis, and chronic thromboembolic pulmonary hypertension: a scientific statement from the American Heart Association. Circulation. 2011;123:1788-830. https://doi.org/10.1161/ CIR.0b013e318214914f.

49. Baig S, Bert J, Gertner E. Safety of retrievable inferior vena cava filters in patients with the antiphospholipid syndrome. Eur J Rheumatol. 2018;5. https://doi.org/ 10.5152/eurjrheum.2018.17091

This recent article described a series of cases in which patients with antiphospholipid syndrome underwent IVCF placement and did not experience complications from the temporarily indwelling filters. These findings suggest that the previous strict avoidance of IVCFs in this patient population may be reconsidered.

50. Cherian J, Gertner E. Recurrent pulmonary embolism despite inferior vena cava filter placement in patients with the antiphospholipid syndrome. J Clin Rheumatol. 2005;11:56-8. https://doi.org/10.1097/ 01.rhu.0000152150.01274.1b.

51. Goodin A, Han Q, Raissi D, Brown JD. A review of interventions to increase vena cava filter retrieval rates. Ann Vasc Surg. 2018;51:284-97. https://doi.org/10. 1016/j.avsg.2018.02.010.

52. Klinken S, Humphries C, Ferguson J. Establishment of an inferior vena cava filter database and interventional radiology led follow-up - retrieval rates and patients lost to follow-up. J Med Imaging Radiat Oncol. 2017;61:630-5. https://doi.org/10.1111/1754-9485. 12611.

53. Juluru K, Elnajjar P, Shih H-H, Hiestand B, Durack JC. An informatics approach to facilitate clinical management of patients with retrievable inferior vena cava filters. Am J Roentgenol. 2018;211:W178-84. https:// doi.org/10.2214/AJR.18.19561.

54. Mikhael B, Albaghdadi M, Abtahian F, MacKay C, Secemsky E, Jaff MR, et al. Usefulness of a computerized reminder system to improve inferior vena cava filter retrieval and complications. Am J Cardiol. 2019;123:348-53. https://doi.org/10.1016/j.amjcard. 2018.09.040

55.• Dake MD, Murphy TP, Krämer AH, Darcy MD, Sewall $\mathrm{LE}$, Curi MA, et al. One-year analysis of the prospective 
multicenter SENTRY clinical trial: safety and effectiveness of the Novate Sentry bioconvertible inferior vena cava filter. J Vasc Interv Radiol. 2018;29:1350-

1361.e4. https://doi.org/10.1016/j.jvir.2018.05.009

This recent study discusses the most recent findings regarding the Novate Sentry Bioconvertible IVCF.

56. Eggers MD, McArthur MJ, Figueira TA, Abdelsalam ME, Dixon KP, Pageon LR, et al. Pilot in vivo study of an absorbable polydioxanone vena cava filter. J Vasc Surg Venous Lymphat Disord. 2015;3:409-20. https://doi. org/10.1016/j.jvsv.2015.03.004.

57. Achaibar K, Waldmann C, Taccone FS. The Angel catheter for the prevention of pulmonary embolism: combining an IVC filter and a triple-lumen central venous catheter. Expert Rev Med Devices. 2019;16:183-6. https://doi.org/10.1080/17434440. 2019.1583556.

\section{Publisher's Note}

Springer Nature remains neutral with regard to jurisdictional claims in published maps and institutional affiliations. 